\title{
Towards larger spatiotemporal scales in polymer simulations
}

\author{
LI YanChun ${ }^{1}$, WANG YongLei ${ }^{1}$, LI ZhanWei ${ }^{2}$, LIU Hong $^{1} \&$ LÜ ZhongYuan ${ }^{1 *}$ \\ ${ }^{1}$ State Key Laboratory of Theoretical and Computational Chemistry, Institute of Theoretical Chemistry, Jilin University, Changchun 130021, \\ China; \\ ${ }^{2}$ State Key Laboratory of Polymer Physics and Chemistry, Institute of Applied Chemistry, Chinese Academy of Sciences, Changchun 130022, \\ China
}

Received April 11, 2013; accepted May 6, 2013; published online July 22, 2013

\begin{abstract}
Molecular dynamics simulations are useful tools to unveil molecular mechanisms of polymer phase separation, self-assembly, adsorption, and so on. Due to large molecular size and slow relaxation of the polymer chains, a great amount of issues related to large-distance chain displacement cannot be tackled easily with conventional molecular dynamic simulations. Systematic coarse-graining and enhanced sampling methods are two types of improvements that can boost spatiotemporal scales in polymer simulations. We present two typical ways to obtain the coarse-graining potential either by fitting to correct liquid structures or by fitting to available thermodynamic properties of polymer systems. The newly proposed anisotropic coarse-grained particle model can be used to describe aggregation and assembly of polymeric building blocks from disk-like micelles to Janus particles. We also present a stochastic polymerization model combined with coarse-grained simulations to investigate the problems strongly influenced by the coupling of polymerization and excluded volume effects. Finally, a facile implementation of integrated tempering sampling method is illustrated to be very efficient on bypassing local energy minima and having access to true equilibrium polymer structures.
\end{abstract}

molecular dynamics simulation, spatiotemporal scale, polymer simulation

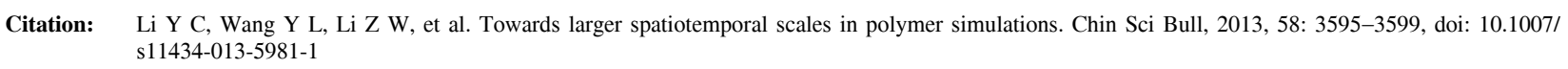

Molecular dynamics (MD) is a powerful simulation technique proven to produce highly realistic results in a wide variety of applications over the past decade $[1,2]$. However, the computational costs of detailed interaction models in this paradigm severely limit its applicability beyond extremely small spatiotemporal scales. In fields such as polymer and molecular biology, many interesting phenomena occur in length and time scales much bigger than those pertaining to the motion of single atom or molecule. Hence both coarse-grained MD and dissipative particle dynamics (DPD) techniques $[3,4]$ were extensively developed and used in order to allow simulations of complex soft matter systems in larger scales more relevant to these processes [5-9], by omitting degrees of freedom not immediately essential for the description of systems at studied level

*Corresponding author (email: luzhy@jlu.edu.cn)
[10-13]. For slowly-evolving polymer systems, it is also of great interest to resort to enhanced sampling techniques developed recently for approaching better thermodynamic average calculations [14-17]. Therefore, by combining both systematic coarse-graining and enhanced sampling methods, it is possible to investigate polymer systems with extended spatiotemporal scales that are much relevant in experiments but inaccessible with conventional atomistic detailed MD simulations.

\section{Coarse-graining methods}

\subsection{Coarse-graining method based on structure infor- mation}

For a better understanding of intrinsic properties of particular polymer systems, both intermolecular and intramolecular 
interaction information and chemical compositions are necessary. One way to access that is to develop particular coarse-grained (CG) model by grouping all atoms of a molecule into several interacting beads. The construction of effective interaction parameters between $\mathrm{CG}$ beads is the subsequent major challenge, for which the main philosophy is to accurately describe the physical and chemical properties with an effective potential as simple as possible [18-20].

Chen et al. [21] proposed an automatic coarse-graining method and applied it on polyethylene (PE) system. The CG potential $F(r)$ was equivalent to the free energy due to the integration of degrees of freedom by coarse-graining,

$$
F(r)=-k_{\mathrm{B}} T \ln g(r),
$$

where the radial distribution function (RDF) $g(r)$ between the CG beads could be obtained either from atomistic MD simulations or from experiments. The trial potentials could be renewed via an iterative equation

$$
F_{\text {new }}(r)=F_{\text {old }}(r)+f k_{\mathrm{B}} T \ln \left[g(r)_{\text {trial }} / g(r)_{\text {target }}\right],
$$

where $f$ is a constant. In [21], $g(r)_{\text {target }}$ was obtained by atomistic MD simulations and $g(r)_{\text {trial }}$ was obtained from Lowe-Andersen simulations [22]. The trial potential was updated according to eq. (2); and when $g(r)_{\text {trial }}$ was converged to $g(r)_{\text {target }}$ after several iterations, the coarse-grained numerical potential was obtained.

The CG model of PE was obtained by collecting several consecutive united atoms into single CG bead, which was further connected to neighboring CG beads with harmonic springs. Corresponding radial distribution functions with varied coarse-graining levels were calculated from atomistic simulations. At low coarse-graining level, coarse-grained beads exhibited a greater excluded volume effect, which leads to related potentials with strong repulsion between $\mathrm{CG}$ beads at short distance. While at high coarse-graining level, the molecular detail was lost too much. Only at medium coarse-graining level, the CG beads are "soft" enough to allow a larger integration time step in CG simulations while keeping acceptable molecular detail, thus balancing the computation efficiency and accuracy. Subsequently, corresponding numerical CG potentials were obtained by fitting such RDFs. The tabulated effective potentials, instead of conservative potentials applied in CG simulations with Lowe-Andersen thermostat [22], were renewed iteratively until corresponding RDFs are consistent with the ones obtained beforehand. With these derived numerical potentials, larger PE systems could be fully equilibrated in short time under same thermodynamic conditions. Moreover, by using Rosenbluth sampling technique [2], atomistic details could be filled into $\mathrm{CG}$ beads in equilibrated system. Since the polymer chains were produced from equilibrium state, they might be further used in atomistic simulations to study the mechanical properties and so on. This scheme with both coarse-graining and fine-graining procedures might be a promising alternative to successfully achieve the equilibrium state in polymer systems.

\subsection{Coarse-graining method based on thermodynamic properties}

Alternatively in DPD simulations, the effective interaction parameters between $\mathrm{CG}$ beads can also be determined by correctly reproducing thermodynamic properties of real systems [13,23]. Recently, Wang et al. [24] studied the binding structures of charged dendrimers on model biomembranes. Consistent CG models of polyamidoamine dendrimer and dimyristoylphosphatidylcholine molecule were constructed based on equal volume criterion. The newly proposed ENUF-DPD method [25] was adopted to calculate the long-range electrostatic interactions between charged CG beads more precisely and quickly. The short-range and soft interactions between CG beads combined with the long-range electrostatic interactions were used to determine the thermodynamic properties of the dendrimer systems [4]. The conservative interaction parameters of CG beads were related to their chemical identities and solubility data in water. Based on these interaction parameters, CG models of dendrimer and lipid could correctly describe the conformational behavior of dendrimer and properly reproduce the surface tension of amphiphilic
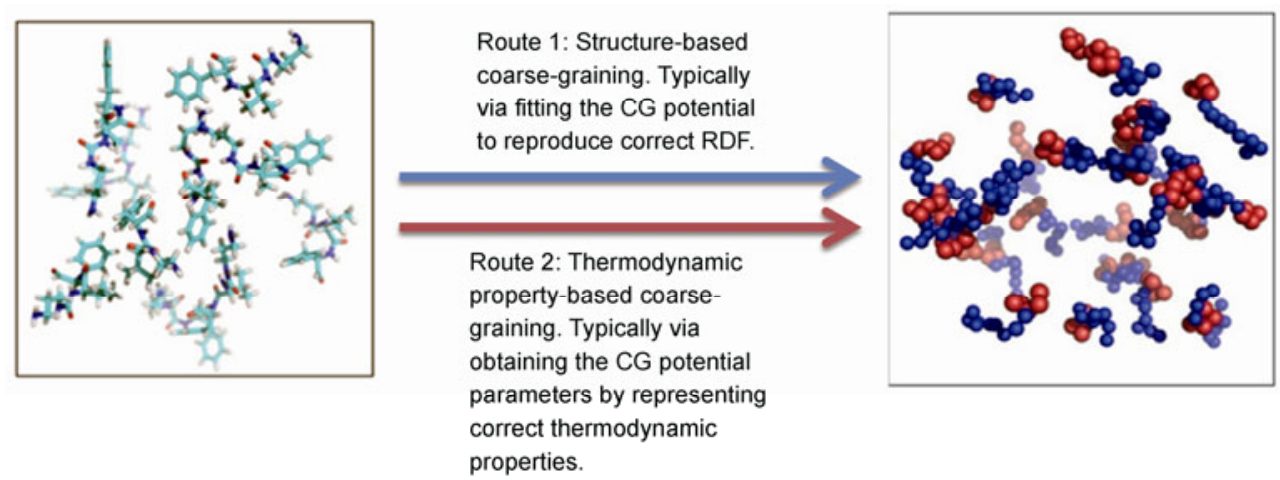

properties.

Figure 1 Two typical routes to build up coarse-grained models from atomistic simulations of polymer chains. 
membrane, respectively.

Small dendrimers were adsorbed preferentially onto the edge of membrane, and were prone to remove lipid molecules from the membrane and expand pores mostly from the edge of existing defects. Large dendrimers strongly resided on the membrane, leading dendrimer-membrane contact regions being characterized with local high surface tension, which would promote the formation of small, isolated holes on membrane and facilitate the transfer of drug molecules to specific targets. Meantime, the permeability of dendrimers across amphiphilic membranes was enhanced by their synergistic effect when dendrimer concentration is increased. These mesoscopic simulation results can provide valuable insight and guidelines for the future synthesis and applications of charged dendrimers as nano-vehicles in gene and drug delivery processes. Similar coarse-graining schemes were also adopted in the studies of the self-assembly of other polymeric materials in dilute solutions and in melts $[13,26,27]$.

\subsection{Describing reptation motion in coarse-grained polymer melts}

For DPD method, the main advantage is that it can describe hydrodynamic behavior of complex fluids correctly. But the bond crossing among polymer chains is inevitable in conventional DPD, which may cause incorrect dynamic behavior of polymer melts. In order to avoid such a limitation, Liu et al. [28] proposed an easy-to-use technique to largely minimize the possibility of bond crossing in DPD simulations. Firstly, a hard core was added to each CG bead so that CG beads could not penetrate each other. Secondly, the spring constant was adjusted to control the maximum bond length. Therefore it is possible to effectively eliminate bond crossing in DPD simulations with a simple geometry constraint. In case the problem of interest is on the length scale of polymer chains instead of polymer segments, it will be convenient to omit all molecular details and take a single polymer chain as a super coarse-grained bead [29]. The interaction between highly coarse-grained beads was described by ultra-soft DPD-type potential. The creation of entanglements between moving beads (chains) was taken as stochastic events on such a high coarse-graining level, while the disappearance of entanglement also obeyed certain rules with designed probabilities. The rates of creation and annihilation of entanglements held a balance to keep the total number of entanglements in polymer melt fluctuating around a constant value. This newly proposed model not only reflects the characteristics of chain entanglements, but also correctly simulates the dynamic properties of polymers, such as the shear viscosity and the mean-square displacement of the chains. Although there are no specific details of molecules, an obvious benefit of this model is the large spatiotemporal scale simulations with much reduced computation cost.

\subsection{Coarse-grained models for anisotropic particles}

In recent years, anisotropic particles with different size, shape and surface functionality had brought an almost unbelievable revolution in materials science [30-34]. The formation of surface functionality on the particles can produce a lot of surface-anisotropic particles, which are often termed as patchy particles and Janus particles [32].

In most cases, patchy particles are described as rigid and non-deformable with fixed particle sizes. Nevertheless, the aggregation and assembly of soft and deformable patchy particles has also attracted increasing interest due to their superior performances and great relevance to applications. Soft patchy particles can be prepared by atom transfer radical polymerization, click chemistry with polymerization, and so on. The modulus of soft patchy particles may be regulated by adjusting the crosslinking density, the grafting density, the number of primary branches and the density of radial branches. Due to the deformability and anisotropic features, they may generate strikingly new self-assembly structures that cannot be obtained with conventional rigid colloidal particles.

In order to study the influencing factors of softness and deformability in assemble process, we presented a simulation model for describing various crosslinking densities in the two-patch particle matrix and/or in the two patches [11]. In our model, the softness and deformability of the patchy particle could be regulated by adjusting the crosslinking densities. For the two-patch particles in bad solvent for patches (while good for the matrix), linear thread-like structures and three-dimensional network structures are obtained at different crosslinking densities, respectively. For the two-patch particles in good solvent for patches (while bad for the matrix), interconnected membrane structures and cluster structures are formed at different crosslinking densities, respectively. Bicontinuous membranes can also be found by adjusting the crosslinking densities of different patches.

Moreover, we suggested an efficient mesoscale model to investigate the assembly of soft Janus particles [35]. The soft Janus particles in our simulations may refer to Janus micelles, Janus microgels and Janus dendrimers that are typically prepared from block copolymers. By properly adjusting Janus balance and attractive interactions between patches, a series of novel hierarchical superstructures are obtained in dilute solutions, such as double helices, single helices, tetragonal bilayers, and so on. Thus, our works demonstrate that soft patchy and Janus particles with deformable and noncentrosymmetric characteristics may provide new possibilities in the fabrication of ordered hierarchical superstructures.

\subsection{Combining polymerization in coarse-grained simulations}

In some cases, the coupling of polymerization process with 
excluded volume effect dominates the physical properties of the polymer systems, including the initialization efficiency, the chain length distribution, and so on. On the length and time scales concerned in this type of problem, atomistic detailed reaction mechanism is irrelevant. We therefore proposed a stochastic reaction model which can be used to study polymerization-induced morphology formation, surface initiated polymerization and so on, in CG simulations [36-39].

In this reaction model, we adopted reaction probability to control the formation of the new bonds. In case an active chain end encounters a number of free monomer beads in the reaction radius, firstly it randomly selects one of the beads as a reacting target. Then another random number $P$ is generated and compared with the preset reaction probability $P_{\mathrm{r}}$ to decide whether the selected bead will be bonded with the active chain or not. Figure 2 schematically illustrates this stochastic polymerization model.

With this simple reaction model, we studied, for example, the influence of surface curvature on surface initiated polymerization (SIP) processes. We found that, depending on different criteria for ceasing the reaction, different relationships between grafted chain polydispersity index (PDI) and the grafting surface curvature can be categorized [39]. Some previous speculations that the concave SIP seems to lead to general increase in PDI with increasing surface curvature, may fail to generalize the true dependence of PDI on the surface geometry. Besides, our results also showed that the enhanced confinement in concave SIP yields the decrease of molecular weight, which is consistent with experimental results. These results shed light on better control and design of functional porous materials for use in bioimplanting or chemical sensors.

\section{Enhanced sampling with ITS}

Because the free energy landscape of typical polymeric system is rough and complicated with plenty of minima and barriers, it is difficult to search global free energy minimum

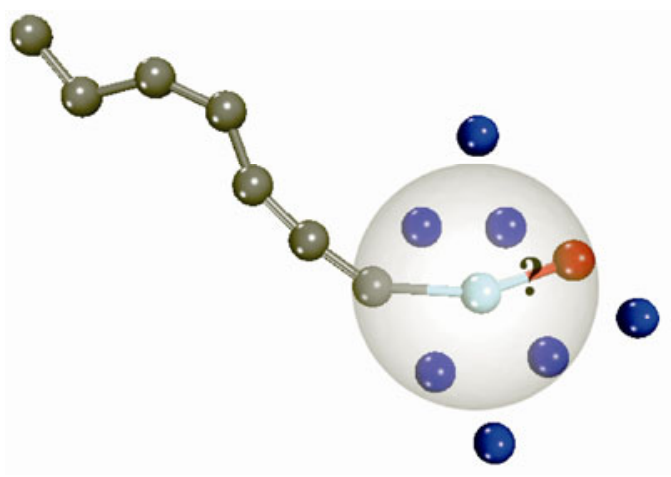

Figure 2 Illustration of the reaction model and the concept of reaction probability. Active chain: gray, active end: cyan, reacting target: red. using conventional (even coarse-grained) MD or MC simulations. With the help of recently developed enhanced sampling methods, such as integrated tempering sampling (ITS) method, we can efficiently bypass local energy minima and have access to true equilibrium polymer structures.

ITS method is based on the generalized ensemble to get a distribution covering a broad range of energies. We define the generalized distribution function $W(r)$ as a summation of a set of Boltzmann factors at different temperatures $T_{k}$ :

$$
W(r)=\sum_{k} n_{k} e^{-\beta_{k} U(r)}, \quad k=1,2, \cdots N,
$$

where $\beta_{k}=1 / k_{\mathrm{B}} T_{k}$. The weighting factor $n_{k}$ is a positive constant related to the contribution of the temperature $T_{k}$. Thermodynamic properties at temperature $T, T \in\left[T_{1}, T_{N}\right]$, can be evaluated by reweighting the ensemble averages obtained in generalized ensemble simulations. The key issue in ITS method is to determine these weighting factors together with suitable temperature range and spacing. We proposed an efficient way to obtain parameters such as the set of temperatures and the corresponding weighting factors from canonical average of potential energies [17]. These parameters can be easily obtained without estimating partition functions.

This ITS method was then used in identifying the coil-globule transition of a flexible polymer chain. In a single ITS simulation composing 18 temperatures ranging from 1.0 to 4.35 , we accurately obtained the dependence of chain radius of gyration on the temperature, by which we could easily identify the coil-globule transition point for a sufficient long polymer chain [17]. ITS method is at least one order of magnitude faster than conventional MD on coping with this type of problem.

\section{Conclusions}

In summary, we argue that the systematic coarse-graining together with enhanced sampling methods are two types of versatile tools towards larger spatiotemporal scales in polymer simulations. Combining both methods in GPU accelerated simulation toolkits will eventually enhance our ability to solve more experimentally relevant problems by computer simulations.

This work was supported by the National Basic Research Program of China (2012CB821500) and the National Natural Science Foundation of China (21025416, 50930001).

1 Allen M P, Tildesley D J. Computer Simulation of Liquids. Oxford: Oxford University Press, 1987

2 Frenkel D, Smit B. Understanding Molecular Simulation. San Diego: Academic Press, 1996

3 Hoogerbrugge P J, Koelman J M V A. Simulating microscopic hydrodynamic phenomena with dissipative particle dynamics. Europhys Lett, 1992, 19: 155-160

4 Groot R, Warren P. Dissipative particle dynamics: Bridging the gap 
between atomistic and mesoscopic simulation. J Chem Phys, 1997, 107: 4423-4435

5 Qian H J, Lu Z Y, Chen L J, et al. Computer simulation of cyclic block copolymer microphase separation. Macromolecules, 2005, 38: 1395-1401

6 Liu W, Qian H J, Lu Z Y, et al. Dissipative particle dynamics study on the morphology changes of diblock copolymer lamellar microdomains due to steady shear. Phys Rev E, 2006, 74: 021802

7 He Y D, Qian H J, Lu Z Y, et al. Polymer translocation through a nanopore in mesoscopic simulations. Polymer, 2006, 48: 3601-3606

8 Qian H J, Chen L J, Lu Z Y, et al. Surface diffusion dynamics of a single polymer chain in dilute solution. Phys Rev Lett, 2007, 99: 068301

9 Liu Y T, Zhao Y, Liu H, et al. Spontaneous fusion between the vesicles formed by $\mathrm{A}_{2 n}\left(\mathrm{~B}_{2}\right)_{n}$ type comb-like block copolymers with semiflexible hydrophobic backbone. J Phys Chem B, 2009, 113: 1525615262

10 Zhang J, Lu Z Y, Sun Z Y. A simple route to fabricate patchy nanoparticle via self-assembly of a multiblock copolymer chain in one step. Soft Matter, 2011, 7: 9944-9950

11 Zhang J, Lu Z Y, Sun Z Y. Self-assembly of amphiphilic patchy particles with different cross-linking densities. Soft Matter, 2012, 8: 7073-7080

12 Zhang J, Lu Z Y, Sun Z Y. Self-assembly structures of amphiphilic multiblock copolymer in dilute solution. Soft Matter, 2013, 9: 1947-1954

13 Liu Y, Yu C, Jin $\mathrm{H}$, et al. A supramolecular Janus hyperbranched polymer and its light-reversible self-assembly of narrowly-distributed vesicles. J Am Chem Soc, 2013, 135: 4765-4770

14 Sugita Y, Okamoto Y. Replica-exchange molecular dynamics method for protein folding. Chem Phys Lett, 1999, 314: 141-151

15 Wang F, Landau D P. Efficient, multiple range random walk algorithm to calculate the density of states. Phys Rev Lett, 2001, 86: 2050-2053

16 Gao Y Q. An integrate-over-temperature approach for the enhanced sampling in molecular dynamics simulations. J Chem Phys, 2008, 128: 064105

17 Zhao P, Yang L J, Gao Y Q, et al. Facile implementation of integrated tempering sampling method to enhance the sampling over a broad range of temperatures. Chem Phys, 2013, 415: 98-105

18 Lyubartsev A P, Laaksonen A. Calculation of effective interaction potentials from radial distribution functions: A reverse Monte Carlo approach. Phys Rev E, 1995, 52: 3730-3737

19 Müller-Plathe F. Coarse-graining in polymer simulation: From the atomistic to the mesoscopic scale and back. Chem Phys Chem, 2002, 3: 754-769

20 Baschnagel J, Binder K, Doruker P, et al. Bridging the gap between atomistic and coarse-grained models of polymers: Status and perspectives. Adv Polym Sci, 2000, 152: 41-156

21 Chen L J, Qian H J, Lu Z Y, et al. An automatic coarse-graining and fine-graining simulation method: Application on polyethylene. J Phys Chem B, 2006, 110: 24093-24100

22 Chen L J, Lu Z Y, Qian H J, et al. The effects of Lowe-Andersen temperature controlling method on the polymer properties in mesoscopic simulations. J Chem Phys, 2005, 122: 104907

23 Zhao Y, Lu Z Y, Sun C C. Dissipative particle dynamics study of the multicompartment micelles self-assembled from the blend of diblock copolymer poly(ethyl ethylene)-block-poly(ethylene oxide) and homopolymer poly(propylene oxide) in aqueous solutions. Polymer, 2009, 50: 5333-5340

24 Wang Y L, Lu Z Y, Laaksonen A. Specific binding structures of dendrimers on lipid bilayer membranes. Phys Chem Chem Phys, 2012, 14: 8348-8359

25 Wang Y L, Laaksonen A, Lu Z Y. Implementation of non-uniform FFT based Ewald summation in dissipative particle dynamics method. J Comput Phys, 2013, 235: 666-682

26 Moeinzadeh S, Jabbari E. Mesoscale simulation of the effect of a lactide segment on the nanostructure of star poly(ethylene glycol-co-lactide)-acrylate macromonomers in aqueous solution. J Phys Chem B, 2012, 116: 1536-1543

27 Soto-Figueroa C, Rodríguez-Hidalgo M, Martínez-Magadán J. Dissipative particle dynamics study of order-order phase transition of BCC, HPC, OBDD, and LAM structures of the poly(styrene)poly (isoprene) diblock copolymer. Macromolecules, 2008, 41: 32973304

28 Liu H, Xue Y H, Qian H J, et al. A practical method to avoid bond-crossing in two dimensional dissipative particle dynamics simulations. J Chem Phys, 2008, 129: 024902

29 Zhu Y L, Liu H, Lu Z Y. A highly coarse-grained model to simulate entangled polymer melts. J Chem Phys, 2012, 136: 144903

30 Glotzer S C. Some assembly required. Science, 2004, 306: 419-420

31 Glotzer S C, Solomon M J. Anisotropy of building blocks and their assembly into complex structures. Nat Mat, 2007, 6: 557-562

32 Pawar A B, Kretzschmar I. Fabrication, assembly, and application of patchy particles. Macromol Rapid Commun, 2010, 18: 150-168

33 Li Z W, Sun Z Y, Lu Z Y. A simulation model for the hierarchical self-assembly of soft disklike micelles. J Phys Chem B, 2010, 114: 2353-2358

34 Jia X X, Li Z W, Sun Z Y, et al. Hierarchical self-assembly of soft disklike particles under shear flow. J Phys Chem B, 2011, 115: 13441-13448

35 Li Z W, Lu Z Y, Sun Z Y, et al. Model, self-assembly structures, and phase diagram of soft Janus particles. Soft Matter, 2012, 8: 26932697

36 Liu H, Qian H J, Zhao Y, et al. Dissipative particle dynamics simulation study on the binary mixture phase separation coupled with polymerization. J Chem Phys, 2007, 127: 144903

37 Liu H, Li M, Lu Z Y, et al. Influence of surface-initiated polymerization rate and initiator density on the properties of polymer brushes. Macromolecules, 2009, 42: 2863-2872

38 Liu H, Li M, Lu Z Y, et al. A multiscale simulation study on the curing reaction and the network structure in typical epoxy system. Macromolecules, 2011, 44: 8650-8660

39 Liu H, Zhu Y L, Zhang J, et al. Influence of concave surface curvature on chain polydispersity in surface-initiated polymerization. ACS Macrolett, 2012, 1: 1249-1253

Open Access This article is distributed under the terms of the Creative Commons Attribution License which permits any use, distribution, and reproduction in any medium, provided the original author(s) and source are credited. 